\title{
THE EXTRAGALACTIC GAMMA-RAY SKY
}

\section{A VIEW ON THE MOST POWERFUL PHENOMENA IN THE UNIVERSE}

\section{MONICA ORIENTI}

The gamma-ray sky provides a look into the most energetic and violent processes of the universe. In the last years, gamma-ray satellites are scanning the sky for understanding the physics governing the gamma-ray emission. A wealth of information on the physics of the gamma-ray sky has been obtained by gamma-ray satellites orbiting around the Earth. So far, roughly three thousands sources have been detected in gamma-rays, and this number is going to increase as the gamma-ray missions continue to survey the sky. The high energy sky is dominated by extragalactic objects. A large fraction of the detected sources has no obvious counterpart in catalogs at other wavelengths, leaving their nature under debate. This contribution will focus on the extragalactic gamma-ray sky, its properties and processes at work with the aim of discussing the main issues highly debated in the astrophysical community.

Keywords: gamma-rays, extragalactic astronomy, non-thermal mechanisms, active galaxies, quasars.

THE GAMMA-RAY ASTROPHYSICS: AN EYE ON THE ENERGETIC UNIVERSE

During a crystal clear night, stars, planets, and galaxies fill the sky with their light. What we can see with our bare eyes is only a small fraction of what celestial bodies emit. The radiation produced by astrophysical processes covers a huge range of energies. The terrestrial atmosphere is opaque to the majority of the electromagnetic spectrum, and two small windows, in radio and optical bands, permit the light at such wavelengths to pass through and reach the ground.

To deal with this observational limitation, telescopes for infrared, ultraviolet, $\mathrm{X}$-ray, and gamma-ray observations have been placed on board satellites orbiting around the Earth. The gamma-ray radiation represents the most energetic «light» of the electromagnetic spectrum. Gamma-ray photons have energies above a few $\mathrm{MeV}^{1}$ and they can be

$1 \mathrm{eV}$ : electronvolt, energy acquired by an electron when it goes across an electric potential difference of one Volt. as high as $100 \mathrm{TeV}$ or even more. Such high energies are produced by some of the most powerful and violent processes taking place in the universe.

Gamma-ray emitters are found at all scales: the Moon and the flaring Sun in our Solar System, supernova remnants, high-mass binaries, and pulsars in our galaxy, and relativistic jets produced by supermassive black holes hosted in active galaxies at cosmological distances. These discrete sources are superimposed to a diffuse radiation made by two main components: one permeates the galactic plane and is produced by interactions of cosmic rays with the interstellar matter and radiation field of the Milky Way, while the other is isotropically distributed in the sky, probably of extragalactic origin, and may be the collective glow of faint sources that are not detected singularly.

Although gamma-ray emission from the interstellar space was predicted in the late 1940s (e.g., Feenberg \& Primakoff, 1948), the first identification of high-energy cosmic gamma rays from galactic and extragalactic sources occurred 
in the 1960s by the MIT gamma-ray instrument on board the Third Orbiting Solar Observatory (OSO 3) (Kraushaar et al., 1972). Then, the European satellite COS-B detected photons in the $50-500 \mathrm{MeV}$ range from the quasar 3C 273 (Swanenburg et al., 1978). However, we had to wait for the Energetic GammaRay Experiment Telescope (EGRET) on board the Compton Gamma-Ray Observatory (CGRO) in the 1990s for a complete survey of the gamma-ray sky. EGRET observed the sky between $30 \mathrm{MeV}$ and about $20 \mathrm{GeV}$ for almost a decade, and disclosed the variable and variegated character of the high-energy sky. EGRET detected nearly 300 gamma-ray sources above $100 \mathrm{MeV}$, but only a small fraction ( 30\%) was identified as the high-energy counterpart of known objects observed at other wavelengths. The large majority of identified sources had an extragalactic origin (Hartman et al., 1999).

Since 2007 we have been experiencing a golden age of the gamma-ray astrophysics. The launch of the Italian satellite AGILE followed a year after by the advent of the Large Area Telescope (LAT) on board the Fermi satellite renewed the interest of the astrophysical community in the gamma-ray sky. Despite the nonuniform sky coverage, during its first year of observation AGILE detected roughly 50 high-confidence gamma-ray sources (Pittori et al., 2009). On
«SINCE 2007 WE HAVE BEEN EXPERIENCING A GOLDEN AGE OF THE GAMMA-RAY ASTROPHYSICS. THE LAUNCH OF THE ITALIAN SATELLITE AGILE WAS FOLLOWED A YEAR AFTER BY THE ADVENT OF THE LAT ON BOARD THE FERMI SATELLITE» is observable just because they are very close to us. mainly surveying the sky, and completes an all-sky map every three hours. With its broad energy range, from $20 \mathrm{MeV}$ to a few $\mathrm{TeV}$, and the improved sensitivity, Fermi-LAT detected about two hundred gamma-ray sources in only three months (Abdo et al., 2009). After four years of observations, Fermi-LAT detected more than 3,000 gamma-ray sources, and discovered high-energy emission from classes of sources which had not been detected before. The majority of the associated gamma-ray sources (about $60 \%$ ) have an extragalactic nature, while about 250 objects are from our galaxy, pulsars being the largest galactic source class (Acero et al., 2015).

These numbers may look tiny if compared to the hundreds of thousand or even hundreds of million objects listed in radio, infrared, optical and X-rays catalogs. We must keep in mind that not all the celestial bodies are strong gamma-ray emitters. For example, the emission from the Sun, or the Moon, Emission from stars and interstellar dust and gas is easy to see in infrared, optical, ultraviolet and X-rays, while their contribution in the high-energy band is below the detectability of the current telescopes.

This review is focused on the gamma-ray emission from the extragalactic sky and deals with the various source populations detected by gamma-ray telescopes so far. I will discuss the complex task of finding the low-energy counterpart of a gamma-ray source, and the critical role that multiband observations play in providing a firm source identification. Finally, a brief overview of the very high energy sky and of the next generation of gamma-ray telescopes will be presented.

\section{THE EXTRAGALACTIC GAMMA-RAY SKY}

Galaxies are the main bricks of the universe. Stars, gas, and dust are responsible for the galaxy emission across the electromagnetic spectrum, including gamma rays which are produced by the interaction of cosmic ray particles, accelerated for example by supernovae blasts, with the interstellar gas. However, this emission is very faint and only in Andromeda and in the Large and Small Magellanic Clouds has been detected so far.

In addition to the three galaxies in the Local Group, the Milky Way, Andromeda and the

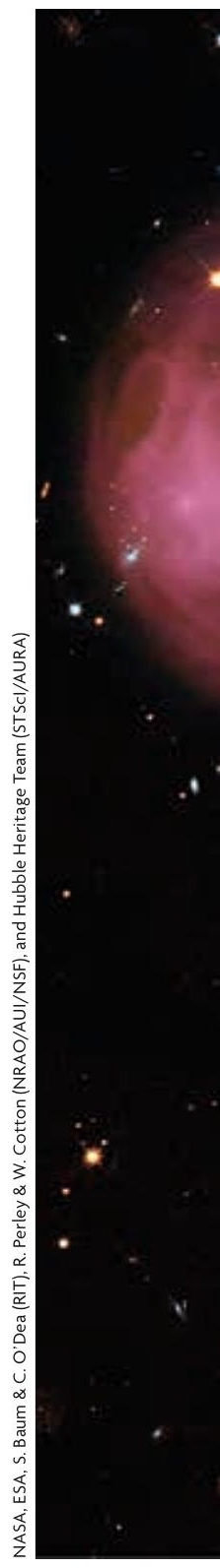

Triangulum galaxy, Fermi-LAT detected high-energy emission from a handful of starburst galaxies. In these galaxies the rate at which stars are produced (and explode) is much higher than in galaxies like the Milky Way. This is reflected in a high rate of supernovae explosions and thus of cosmic-rays, which are at the basis of the gamma-ray emission.

Normal galaxies and starbursts are orders of magnitude less luminous than active galaxies. About $10 \%$ of galaxies host an active nucleus at their centre. Active galactic nuclei are the manifestation of the extraordinary amount of energy released by a supermassive black hole (Blandford, Netzer, \& Woltjer, 1990). In about $10 \%$ of active galactic nuclei, the supermassive black hole develops and launches a bipolar outflow of relativistic particles which may extend well beyond the host galaxy (Figure 1). At the edge of the two jets, relativistic particles interact 


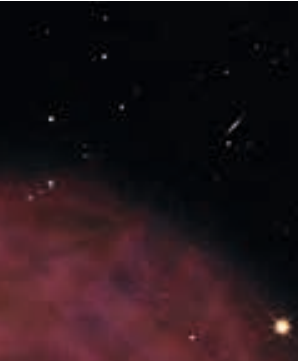

$\therefore$
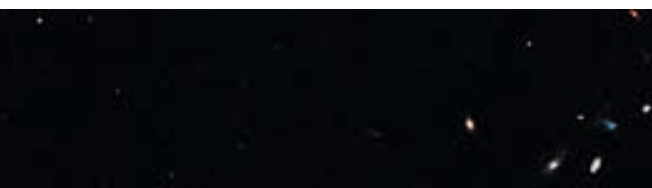

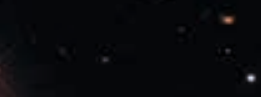
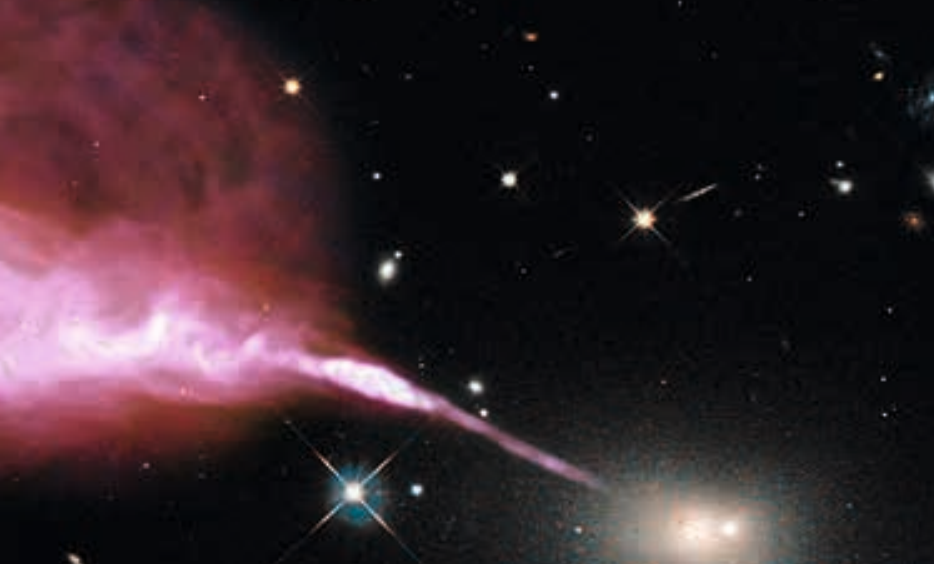

8

3
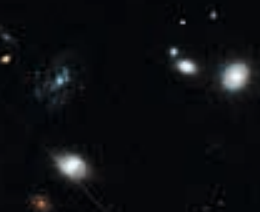

$*$

$+2$

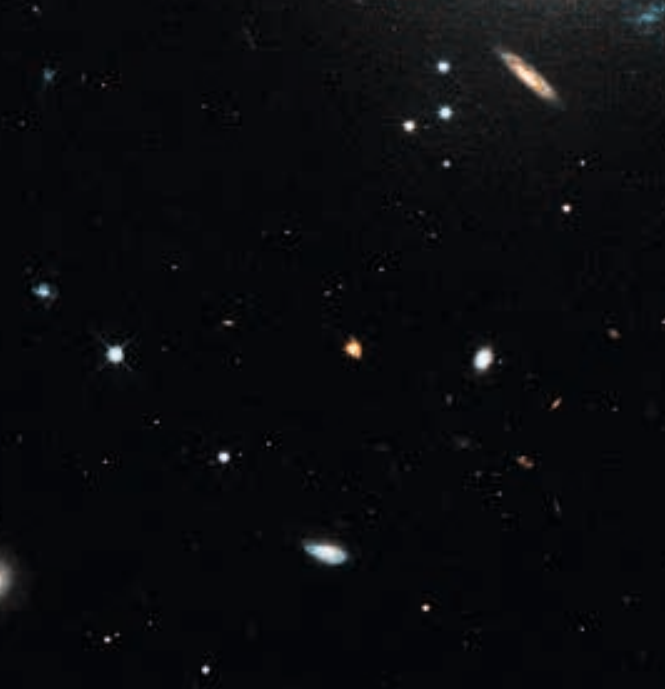

Figure 1. Radio emission (in pink) overlaid on an optical image. The radio emission from powerful radio sources extends much beyond the host galaxy in which the relativistic jets originate.

\section{"STARS, GAS, AND DUST ARE \\ RESPONSIBLE FOR THE GALAXY \\ EMISSION ACROSS THE \\ ELECTROMAGNETIC SPECTRUM, \\ INCLUDING GAMMA RAYS"}

with the surrounding medium and form spectacular structures of diffuse emission.

Relativistic particles lose energy as they interact with the magnetic fields of the jet and produce synchrotron radiation visible from radio up to $\mathrm{X}$-rays. Furthermore, relativistic particles interact also with the surrounding radiation fields. During this interaction, the relativistic particles transfer part of their energy to the less energetic photons. As a consequence, the photons gain energy, eventually becoming gamma-ray photons. This scatter process is called inverse Compton.

In the sub-population of active galactic nuclei known as blazars, the axis of the relativistic jet points 
toward the Earth and its emission is amplified due to relativistic effects (Figure 2). As a consequence these objects look brighter than what they are, facilitating their detection. The luminosity of blazar objetcs ranges, on average, between $10^{45} \mathrm{erg} / \mathrm{s}^{2}$ and $10^{47} \mathrm{erg} / \mathrm{s}$, but it can reach, in a few cases, values as high as $10^{50} \mathrm{erg} / \mathrm{s}$. Furthermore, every perturbation, like the formation and propagation of a shock, is amplified making the blazar emission highly variable at all wavelengths.

The extragalactic gamma-ray sky seen by FermiLAT is highly dominated by blazars, which represent $98 \%$ of the detected sources. On the other hand, radioloud active galactic nuclei whose relativistic jets point away from the Earth, and for this reason are termed «misaligned objects», do not undergo any luminosity enhancement making their detection at high energies a challenging task. The number of misaligned sources has constantly increased with the improvement of the instrument sensitivity, going from the detection of only Centaurus A by EGRET, up to about 30 objects detected by Fermi-LAT (Ackermann et al., 2015).

\section{"THE POPULATION OF GAMMA- RAY SOURCES HAS DRAMATICALLY INCREASED SINCE THE FIRST DISCOVERY OF A QUASAR IN THE 70s"}

Their gamma-ray luminosity is between $10^{41} \mathrm{erg} / \mathrm{s}$, for the nearby radio galaxy Centaurus A, and $10^{44} \mathrm{erg} / \mathrm{s}$, i.e., at the low-luminosity tail of blazars. However, misaligned objects detected in gamma rays so far are much closer to us with respect to blazars. Since the luminosity decreases as the square of the distance, farther objects must be more luminous in order to be detected. This implies that if a source like Centaurus A were at the average distance of blazars, the observed luminosity would be undetectable by current instruments. Despite being undetectable individually, their cumulative emission contributes to the diffuse gamma-ray background observed almost isotropically in the sky.

\section{IDENTIFIED, ASSOCIATED AND UNASSOCIATED SOURCES: WHAT IS THE MEANING?}

As mentioned in the previous section, active galactic nuclei, and in particular blazars, are the largest class of extragalactic associated sources. The spatial resolution

2 An erg is equivalent to $10^{-7}$ Joules (J).

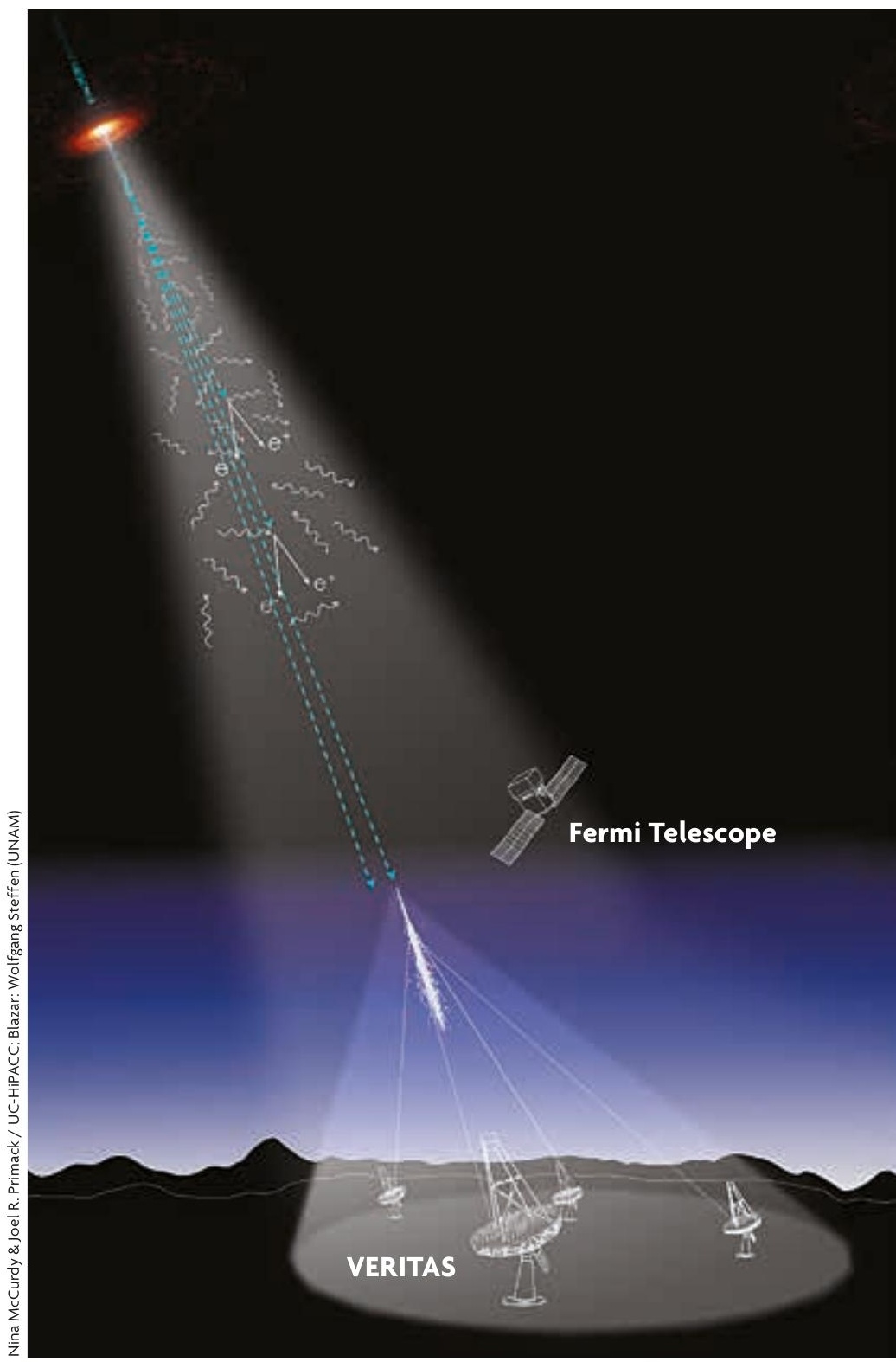

Figure 2. Detection of gamma-ray emission from a blazar. The high energy photons are observed by gamma-ray telescopes orbiting around the Earth, while very-high-energy photons interact with the terrestrial atmosphere and produce particle cascades that are observed by the Cherenkov Telescopes on the ground. Not all the emission from the blazar reaches us because during its path to the Earth it interacts with foreground photons creating particles.

\footnotetext{
"VERY HIGH ENERGY SOURCES OBSERVED SO FAR ARE LIKELY THE TIP OF THE ICEBERG, DUE TO THE OBSERVATIONAL CONSTRAINTS OF THE CURRENT CHERENKOV TELESCOPES»
} 


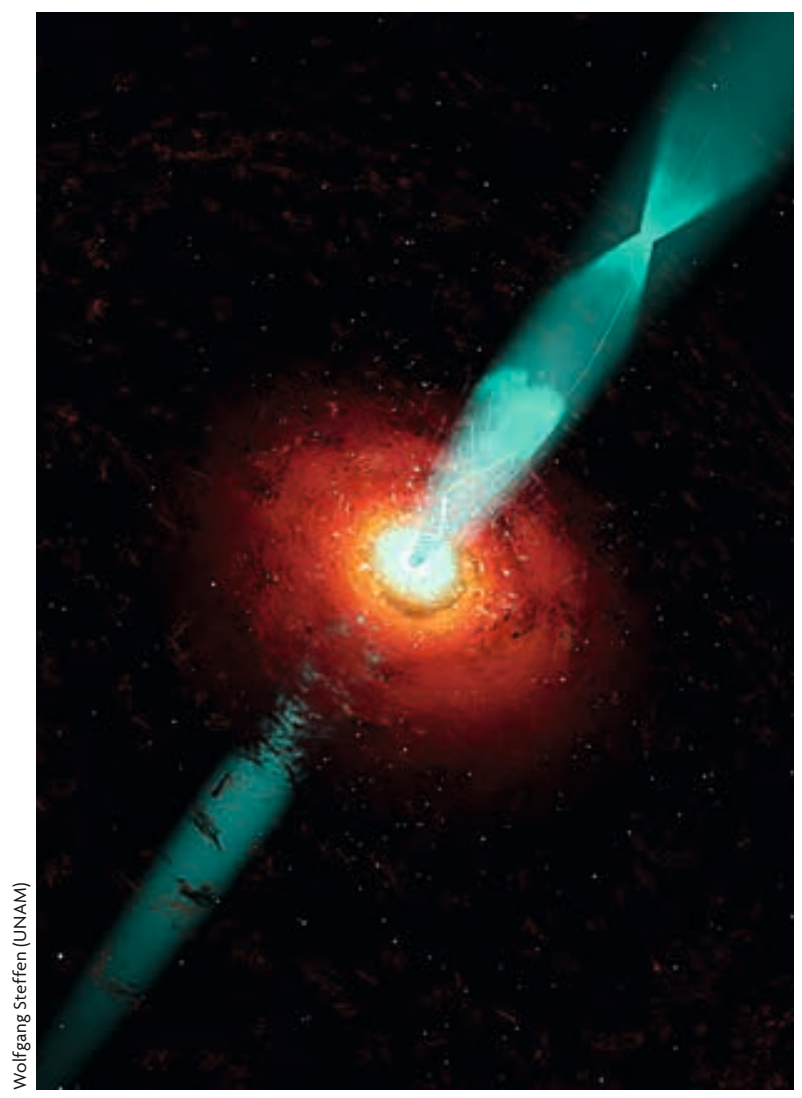

Figura 3. Still from a conceptual animation of $3 \mathrm{C} 120$ created by Wolfgang Steffen. A bipolar outflow of relativistic particles is produced by the super massive black hole at the centre of an active galaxy.

of gamma-ray telescopes is not very accurate. For example Fermi-LAT, which is nowadays the gamma-ray telescope with the best spatial resolution, has a positional uncertainty of about 0.5 degrees at $1 \mathrm{GeV}$, which corresponds roughly the size of the Sun we observe from the Earth. The number of potential counterparts within a typical gamma-ray positional uncertainty region can be huge, from hundreds to several thousands of possible optical/IR counterparts!

Firmly identifying the low-energy counterpart of a gamma-ray source is very difficult. It requires multi-band monitoring observations, which are not usually available. This is the reason why only a minority of gamma-ray sources have an unambiguous identification. For example, among the 3,033 sources detected by Fermi-LAT, only 238 have been firmly identified, 66 out of them are active galactic nuclei.
The process of associating a gamma-ray source with a known object from astronomical catalogs at other wavelengths (mainly optical, radio, X-rays, and infrared) is mainly based on statistical arguments. The catalogs considered for the comparison comprise the classes of objects which are plausible gammaray emitter. The association depends primarily on the close positional correspondence of the high and low energy counterparts. Then the nature of the counterpart is checked. If one of the possible counterpart is a blazar, which is a well-known class of gamma-ray emitters, then that will be the proposed association for the high-energy source.

Nevertheless, a large fraction of objects have no obvious counterpart in other energy bands. In the third EGRET catalog, the unassociated sources were more than a $60 \%$ of the detected gamma-ray sources (Hartman et al., 1999), although they have decreased to a $30 \%$ in the third Fermi-LAT source catalog (Acero et al., 2015).

It is worth noticing that the association procedures rely on the availability of catalogs with high sensitivity and covering a sky area as large as possible. This has been a critical point for the southern hemisphere, where the oceans cover a larger fraction than in the northern one, precluding the construction of observing facilities as numerous as in the north. The same happens for the galactic plane, for which not many catalogs of extragalactic objects are available.

Apart from the aforementioned excess, the unassociated sources are mostly uniformly distributed in the sky suggesting a potential extragalactic origin. With the aim of enlarging the catalogs of plausible counterparts, several campaigns at various wavelengths have been performed and catalogs are still growing as new observations are made.

\section{MULTIFREQUENCY CAMPAIGNS OF VARIABLE ACTIVE GALACTIC NUCLEI}

Variability is a key feature of blazars. Changes in the luminosity are observed at all wavelengths and are related to periods of different activity: quiescent states are interleaved by periods of enhanced activity. Sometimes, abrupt flares occur.

To date, the properties of the high-energy emission have been investigated with the complementary help 
of multi-wavelength monitoring campaigns. The analysis of the light curve, i.e., how the flux at a given frequency changes as a function of time, points out a possible relation between flaring episodes in gamma-rays and the variability observed across the electromagnetic spectrum, suggesting a common origin for the multi-band variability and the highenergy flaring episode (Figure 3).

Despite decades of efforts, many aspects of the high-energy emission and variability are still elusive. The Fermi satellite, with its ability of surveying the entire sky every three hours, is allowing an accurate characterization of the variable high-energy sky. Hundreds of blazars are continuously monitored and flares are caught promptly. The alert of a gammaray outburst is quickly transmitted to telescopes observing in various electromagnetic bands providing a multi-wavelength follow-up as complete as possible.

Important information on the origin and location of the flare comes from the temporal occurrence of the flare in the observed different energy bands. It has been found that gamma-ray flares occur roughly simultaneously in X-ray, optical, and infrared bands, while at radio wavelengths the flare is delayed by a few days/weeks or even several months (Fuhrmann

«THE IMPROVED SENSITIVITY
AND OBSERVATIONAL
STRATEGY OF THE CURRENT
GAMMA-RAY TELESCOPES
ALLOWED A DETAILED
CHARACTERIZATION OF THE
MAIN SOURCE CLASSES
POPULATING THE HIGH-
ENERGY SKY»

light. These superluminal knots are interpreted as the observational manifestation of a moving shock (Marscher \& Gear, 1985).

Neither all the gamma-ray flares have a counterpart at other wavelengths, nor all flares in low energy bands correspond to a gamma-ray outburst. The origin of these uncorrelated flares is still under debate.

\section{A GLANCE AT THE VERY HIGH ENERGY SKY}

Gamma-ray telescopes orbiting around the Earth are sensitive to gamma-rays with energies up to a few hundred GeV. Above this energy it is hard for gamma-ray satellites to detect sources. Fortunately, our atmosphere helps us in detecting such very high energy photons... from the ground! In fact, these photons interact with the atoms and molecules that they encounter along their path through the terrestrial atmosphere. The interaction originates a particle cascade that propagates around the direction of the incoming very high energy photon. These secondary particles are ultrarelativistic and their speed is higher than the speed of light in the atmosphere (of course they are not faster than the light in vacuum, which is the maximum speed at which all matter and electromagnetic waves can travel).

The difference in velocity between the particles and the light causes the emission of radiation, called Cherenkov radiation, which is then detected by Cherenkov telescopes on the ground.

To date, about 200 sources have been detected at very high energy, almost equally divided into galactic, extragalactic, and of unknown origin. The small number of very high energy objects is due to the fact that only a few celestial phenomena can produce such high energies. Furthermore, the current Cherenkov telescopes do not survey the whole sky, but they point at individual objects usually in high activity state following triggers at other wavelengths, and only when there are no observational limitations, such as clouds or full moon. As in the case of the highenergy gamma-ray sky, extragalactic very high energy sources are mainly blazars with only four nearby radio galaxies detected so far.

These sources are all within 7 billion $\left(7 \times 10^{9}\right)$ light years from the Earth. No farther objects have been 
found so far. The lack of detection of distant objects is not due to the sensitivity limitation, but it is mainly related to physical processes occurring at the high energy photons as they travel across the universe. The extragalactic background light consists of photons produced by stars and galaxies across the universe lifetime. As the high-energy photons propagate through the universe, they interact with the photons of the extragalactic background light from ultraviolet to infrared wavelengths. If the energy of the interacting photons is enough, the photons annihilate and create electron-positron pairs (e.g., Gould \& Schreder, 1967). This results in an attenuation of the gamma-ray sources above a critical energy that depends on the distance. The universe is transparent to photons with energy below $20 \mathrm{GeV}$. Then it becomes progressively opaque to higher and higher energies and the visibility distance decreases.

\section{CONCLUDING REMARKS}

The gamma-ray sky sparkles with a few thousand of powerful and variable celestial bodies. The population of gamma-ray sources has dramatically increased since the first discovery of a quasar in the 70s. The improved sensitivity and observational strategy of the current gamma-ray telescopes allowed a detailed characterization of the main source classes populating the high-energy sky. However, many aspects related to the physics involved in these violent processes are still far to be understood. The availability of larger and larger catalogs will enable us to deal with the still open issues on a statistical approach. This aspect will be crucial for our understanding of the very high energy sky.

As discussed in the previous section, very high energy sources observed so far are likely the tip of the iceberg, due to the observational constraints of the current Cherenkov telescopes and their observing strategy. The next generation of ground-based very high energy telescopes is on the way. The Cherenkov Telescope Array is going to be built in the next years with the aim of improving and expanding our knowledge on the very high-energy universe (Actis et al., 2011). The construction will be in two different sites, in La Palma (Spain) for the northern hemisphere, and in Chile for the southern hemisphere. Thanks to the improvement in sensitivity and in the energy coverage, together with the development of new observing strategies, Cherenkov Telescope Array will have a large discovery potential in key areas of astronomy, astrophysics and fundamental physics research. The Cherenkov Telescope Array, together with multiband and multi-messenger observations will tackle important key issues on the physics of high-energy emission. These include the study of the origin of cosmic rays, the investigation of the particle acceleration in relativistc jets, and the inquiry into the ultimate nature of matter and physics beyond the Standard Model, searching for dark matter and effects of quantum gravity. (-)

\section{REFERENCES}

Abdo, A. A., Ackermann, M., Ajello, M., Atwood, W. B., Axelsson, M., Baldini, L., ... Ylinen, T. (2009). Fermi/Large Area Telescope bright gamma-ray source list. The Astrophysical Journal Supplement Series, 183(1), 46-66. doi: 10.1088/0067-0049/183/1/46

Acero, F., Ackermann, M., Ajello, M., Albert, A., Atwood, W. B., Axelsson, M., ... Zimmer, S. (2015). Fermi Large Area Telescope third source catalog. The Astrophysical Journal Supplement Series, 218(2), 23-64. doi: 10.1088/0067-0049/218/2/23

Ackermann, M., Ajello, M., Atwood, W. B., Baldini, L., Ballet, J., Barbiellini, G., ... Zimmer, S. (2015). The third catalog of active galactic nuclei detected by the Fermi Large Area Telescope. The Astrophysical Journal, 810(1), 14-48. doi: 10.1088/0004-637X/810/1/14

Actis, M., Agnetta, G., Aharonian, F., Akhperjanian, A., Aleksić, J., Aliu, E., ... Zychowski, P. (2011). Design concepts for the Cherenkov Telescope Array CTA: An advanced facility for ground-based high-energy gammaray astronomy. Experimental Astronomy, 32(3), 193-316. doi: 10.1007/ s10686-011-9247-0

Blandford, R. D., Netzer, H., \& Woltjer, L. (1990). Active galactic nuclei. Berlin, Heidelberg: Springer-Verlag.

Feenberg, E., \& Primakoff, H. (1948). Interaction of cosmic-ray primaries with sunlight and starlight. Physical Review, 73(5), 449-469. doi: 10.1103/ PhysRev.73.449

Fuhrmann, L., Larsson, S., Chiang, J., Angelakis, E., Zensus, J. A., Nestoras, I., ... Pearson, T. J. (2014). Detection of significant cm to sub$\mathrm{mm}$ band radio and $\gamma$-ray correlated variability in Fermi bright blazars Monthly Notices of the Royal Astronomical Society, 441(3), 1899-1909. doi: $10.1093 / \mathrm{mnras} /$ stu 540

Gould, R. J., \& Schreder, G. (1967). Opacity of the universe to high-energy photons. Physical Review Letter, 16, 252-254. doi: 10.1103/PhysRevLett. 16.252

Hartman, R. C., Bertsch, D. L., Bloom, S. D., Chen, A. W., Deines-Jones, P., Esposito, J. A., ... Dingus, B. L. (1999). The third EGRET catalog of high-energy gamma-ray sources. The Astrophysical Journal Supplement Series, 123(1), 79-202. doi: 10.1086/313231

Kraushaar, W. L., Clark, G. W., Garmire, G. P., Borken, R., Higbie, P., Leong, V., \& Thorsos, T. (1972). High-energy cosmic gamma-ray observations from the OSO-3 satellite. The Astrophysical Journal, 177, 341-363. doi: 10.1086/151713

Marscher, A. P., \& Gear, W. K. (1985). Models for high-frequency radio outbursts in extragalactic sources, with application to the early 1983 millimeter-to-infrared flare of 3C 273. The Astrophysical Journal, 298, 114-127. doi: 10.1086/163592

Marscher, A. P., Jorstad, S. G., D’Arcangelo, F. D., Smith, P. S., Williams, G. G., Larionov, V. M., ... Ryle, W. T. (2008). The inner jet of an active galactic nucleus as revealed by a radio-to-gamma-ray outburst. Nature, 452(7190), 966-969. doi: 10.1038/nature06895

Pittori, C., Verrecchia, F., Chen, A.W., Bulgarelli, A., Pellizzoni, A., Giuliani, A., ... Salotti, L. (2009). First AGILE catalog of highconfidence gamma-ray sources. Astronomy \& Astrophysics, 506, $1563-$ 1574. doi: 10.1051/0004-6361/200911783

Swanenburg, B. N., Hermsen, W., Bennett, K., Bignami, G. F., Caraveo, P., Kanbach, G., ... Sacco, B. (1978). COS B observation of high-energy gamma radiation from 3C273. Nature, 275, 298. doi: 10.1038/275298a0

Monica Orienti. Researcher at Institute of Radioastronomy (INAF-IRA) in Bologna (Italy). She got the $\mathrm{PhD}$ at the University of Bologna working on radio emission at high angular resolution from extragalactic sources. 